\title{
Sex differential association of dermatomyositis with Sjögren syndrome
}

\author{
Chia-Chun Tseng MD, Shun-Jen Chang PhD, Wen-Chan Tsai PhD, Tsan-Teng Ou MD, Cheng-Chin Wu MD, \\ Wan-Yu Sung MD, Ming-Chia Hsieh PhD, Jeng-Hsien Yen PhD
}

Cite as: CMAJ 2017 February 6;189:E187-93. doi: 10.1503/cmaj.160783

\begin{abstract}
BACKGROUND: Although dermatomyositis and Sjögren syndrome share serologic autoantibodies and genetic polymorphisms, population data about the incidence of Sjögren syndrome in patients with dermatomyositis is unavailable. We performed a nationwide cohort study to explore the potential relation between dermatomyositis and Sjögren syndrome and, if an association exists,

Dec. 31, 2011. Each patient was matched to, at most, 5 control patients from the National Health Insurance Research Database by age, sex and entry date. Cox regression was used to calculate the hazard ratio (HR) and 95\% confidence interval $(\mathrm{Cl})$ of Sjögren syndrome after adjusting for age, sex, rheumatoid arthritis, systemic lupus erythematosus and systemic sclerosis.
\end{abstract} to elucidate whether it varies by sex.

METHODS: We identified all patients with newly diagnosed dermatomyositis from the Registry of Catastrophic Illness Database in Taiwan between Jan. 1, 1998, and
RESULTS: A total of 1602 patients with dermatomyositis and 7981 control patients were enrolled in the study. There was a positive association of having Sjögren syndrome among patients with dermatomyositis after adjusting for age, sex, rheumatoid arthritis, systemic lupus erythematosus and systemic sclerosis (HR 2.67, 95\% Cl 2.01-3.54). The association was more pronounced in the male cohort (HR 2.69, 95\% Cl 1.19-6.09).

INTERPRETATION: We found a sex differential association of Sjögren syndrome among patients with dermatomyositis independent of age and concomitant autoimmune disease. Further studies are required to determine the clinical importance of this association for both outcomes and therapeutic options.

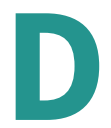

ermatomyositis is an autoimmune disease with multiple dermatologic changes, chronic muscle inflammation, proximal muscle weakness and extramuscular manifestations, including interstitial lung disease. Immunologically, there are several autoantibody specificities, each associated with particular clinical features. Incidence of dermatomyositis is 1.0 per 100000 person-years in British Columbia. ${ }^{1}$ Despite the gains in knowledge of dermatomyositis, the overall mortality is still up to sevenfold higher than that of the general population. ${ }^{2}$ Additionally, those with dermatomyositis have a substantially increased morbidity when compared with the population at large. ${ }^{3}$ The increased risk of malignant disease, ${ }^{2}$ cardiovascular events ${ }^{4}$ and interstitial lung disease $\mathrm{e}^{5}$ in dermatomyositis is well-established. However, the risk of other comorbidities in dermatomyositis has been overlooked. Although an association with Sjögren syndrome has been shown in other autoimmune diseases, including rheumatoid arthritis, ${ }^{6}$ systemic lupus erythematosus ${ }^{7}$ and systemic sclerosis, ${ }^{8}$ data on Sjögren syndrome in patients with dermatomyositis are sparse and mostly limited to case reports. ${ }^{9-11}$
Both dermatomyositis and Sjögren syndrome share common autoantibodies (e.g., anti-poly(U)-binding-splicing factor, ${ }^{12}$ antinuclear antibodies, ${ }^{13,14}$ anti-Sjögren-syndrome-related antigen $A$ [anti-SSA], ${ }^{13,15}$ anti-Sjögren-syndrome-related antigen $B$ [anti$\mathrm{SSB}]^{13,16}$ ) and genetic polymorphisms (e.g., interferon regulatory factor 5 [IRF5 rs4728142]). ${ }^{17,18}$ In addition, patients with Sjögren syndrome have a 4 -fold higher mortality, ${ }^{19}$ with surviving patients incurring a lifetime of increased medical expenses, among other costs. ${ }^{20,21}$

It is important to gain a more accurate understanding of this increased risk of Sjögren syndrome in dermatomyositis. Although research into dermatomyositis showed sex differences in risk factors, ${ }^{22}$ cytokine expression, ${ }^{23}$ autoantibodies profile ${ }^{24}$ and certain dermatomyositis-related outcomes, ${ }^{2}$ it is unknown whether the association with Sjögren syndrome differs among men and women with dermatomyositis.

We undertook this study to determine the incidence of Sjögren syndrome in dermatomyositis when compared with control patients in a general population and examined whether the association between dermatomyositis and Sjögren syndrome varies by sex. 


\section{Methods}

\section{Data source}

In 1995, Taiwan launched the National Health Insurance (NHI) program, with a coverage rate of more than $99.6 \% .{ }^{25}$ The $\mathrm{NHI}$ medical claims database includes data on outpatient, inpatient and dental services. Diseases are classified according to International Classification of Diseases, 9th Revision, Clinical Modification (ICD-9) diagnostic criteria. This study used 2 databases: the National Health Insurance Research Database (NHIRD) and the Registry of Catastrophic Illness. The Registry of Catastrophic Illness is a subpart of the original NHIRD in which patients with catastrophic illness are registered. The NHIRD data set consisted of a randomly selected 1000000 patients from all beneficiaries in the original NHIRD. This representative national sample has comparable distribution in age, sex or mean insured payroll-related amount between the claimants in the sampled data and the original NHIRD. ${ }^{26}$

Under NHI regulations, insured beneficiaries who have catastrophic diseases, including dermatomyositis and Sjögren syndrome, can apply for registration in the Registry of Catastrophic Illness. If registration is approved, copayment is waived. Patients with dermatomyositis are registered in the Registry of Catastrophic Illness after their cases are reviewed by specialists, based on clinical presentation, laboratory studies, electromyography results, pathologic reports and fulfillment of Bohan and Peter criteria. ${ }^{27}$ Registration in the Registry of Catastrophic Illness for Sjögren syndrome is approved based on the 2002 American-European Consensus Group Criteria.

For diagnosis accuracy, a routine peer review is conducted within the Bureau of National Health Insurance. The bureau commissions specialists to regularly review medical records by randomly sampling patient charts and imposes fines for false claims. The NHIRD and the Registry of Catastrophic Illness were the sources of data for the comparison group ${ }^{28}$ and for dermatomyositis/Sjögren syndrome, ${ }^{28,29}$ respectively, in previous studies.

For patient privacy, the National Health Research Institutes encrypted all personal identification numbers before releasing the data files for research purposes.

\section{Study cohort}

From Jan. 1, 1998, to Dec. 31, 2011, all patients with a new diagnosis of dermatomyositis (ICD-9 code 710.3) in the Registry of Catastrophic Illness were identified..$^{28}$ Previous studies have validated this approach. ${ }^{28}$ The date on which the patients received a diagnosis of dermatomyositis in the Registry of Catastrophic Illness was set as the entry date for the dermatomyositis cohort. Patients younger than 20 years of age or patients with a diagnosis of Sjögren syndrome before or within 1 year of a dermatomyositis diagnosis were excluded. For the control cohort, no more than 5 patients without dermatomyositis were selected from the NHIRD for each corresponding patient with dermatomyositis. The entry dates for the control patients were matched dates on which they used any medical services. The patients in the dermatomyositis and control cohorts were matched for age, sex and entry date under similar exclusion criteria to ensure that the follow-up time was the same at baseline. Patients with follow-up time of less than 1 year were excluded owing to inadequate duration to assess long-term exposure status. ${ }^{30}$

\section{Outcome measure}

The Registry of Catastrophic Illness ${ }^{29}$ was used to identify patients with Sjögren syndrome (ICD-9 code 710.2). The coding for Sjögren syndrome in the database has been validated. ${ }^{29}$ The follow-up end point was either the date of last visit before Dec. 31, 2011, or the diagnosis date of Sjögren syndrome in the Registry of Catastrophic Illness, whichever came first.

\section{Covariate assessment}

Rheumatoid arthritis (ICD-9 code 714.0), systemic lupus erythematosus (ICD-9 code 710.0) or systemic sclerosis (ICD-9 code $710.1)^{31}$ often overlaps with Sjögren syndrome. Thus, we obtained data on the respective autoimmune diseases based on the ICD-9 code from the Registry of Catastrophic Illness. Previous studies have used a similar approach. ${ }^{29}$

\section{Statistical analysis}

Because patients with Sjögren syndrome preceding or diagnosed within 1 year after a diagnosis of dermatomyositis were excluded, the first year after entry into the cohort was not counted as followup time. Follow-up time was defined as starting 1 year after a dermatomyositis diagnosis. The follow-up period was calculated from 1 year after cohort entry to the end of follow-up.

We first calculated the incidence of Sjögren syndrome (cases per 1000 person-years) among patients with dermatomyositis and control patients stratified by age and sex, and calculated the ageadjusted standardized incidence ratio (IR) and 95\% confidence intervals (Cls) of Sjögren syndrome in the total, male and female populations. Age-adjusted standardized IR is the ratio of the observed cases of Sjögren syndrome among patients with dermatomyositis to the expected cases of Sjögren syndrome among control patients, using the indirect standardization method.

Multivariate Cox regression was used to determine the hazard ratio (HR) of dermatomyositis with $95 \% \mathrm{Cls}$ after adjusting for age, sex and concomitant autoimmune diseases (rheumatoid arthritis, systemic lupus erythematosus and systemic sclerosis). To assess differences in HR for Sjögren syndrome by sex, a parameter representing interaction between sex and dermatomyositis (i.e., male status $\times$ dermatomyositis) was included. The cumulative incidences of Sjögren syndrome among different groups were estimated using Kaplan-Meier analysis, and differences in the full time-to-event distributions between the study groups were compared using log-rank tests. Two-sided $p$ values less than 0.05 were considered significant. All statistical analyses were performed using SAS (version 9.4, SAS Institute).

\section{Ethics approval}

This study was exempt from ethics approval by the Institutional Review Board of Kaohsiung Medical University Hospital (KMUHIRB-EXEMPT(I)-20150051).

\section{Results}

We selected 1602 patients with dermatomyositis from the Registry of Catastrophic Illness and 7981 control patients from NHIRD. The mean age ( \pm standard deviation) of male patients was 49.45 
$( \pm 15.32)$ years in the dermatomyositis group and $48.45( \pm 15.35)$ years in the control group. The mean age ( \pm standard deviation) of female patients was 47.44 ( \pm 14.37 ) years in the dermatomyositis group and 46.86 ( \pm 14.09 ) years in the control group (Table 1). The age distribution between the two groups was the same in both the male $(p=0.9)$ and female $(p=0.9)$ cohorts.

Incidence rate of Sjögren syndrome in dermatomyositis A total of 266 cases of incidental Sjögren syndrome were identified during the follow-up period, 139 in the dermatomyositis group and 127 in the control group. The incidence rate of Sjögren syndrome was 18.37 cases per 1000 person-years in the dermatomyositis group, compared with 3.11 cases per 1000 person-years in the control group. Age-adjusted standardized IR was 5.92 (95\% Cl 3.829.16) (Table 2). When stratified by age, similar trends persisted.

\section{Sex differential incidence rate ratio of Sjögren} syndrome

In the male cohort, age-adjusted standardized IR was 12.37 (95\% Cl 2.68-57.02) (Table 3). In the female cohort, age-adjusted standardized IR was $5.42(95 \% \mathrm{Cl} 3.42-8.57)$ (Table 4), lower than that of the male cohort. Consistent trends of a higher incidence rate ratio of Sjögren syndrome in the male cohort than in the female cohort were noted (Table 3 and Table 4) when stratified by age.

\section{Interactive effects between dermatomyositis and sex}

In the multivariable regression model, dermatomyositis (HR 2.67, $95 \% \mathrm{Cl} 2.01-3.54$ ) in addition to rheumatoid arthritis (HR 3.79, 95\% $\mathrm{Cl} 2.92-4.92$ ), systemic lupus erythematosus (HR 3.97, 95\% Cl 2.995.28) and systemic sclerosis (HR 1.53, 95\% Cl 1.03-2.28) was associated with a higher incidence of Sjögren syndrome after adjusting for

Table 1: Age distribution of patients with dermatomyositis and control patients at baseline

\begin{tabular}{|c|c|c|c|c|}
\hline \multirow[b]{2}{*}{ Characteristic } & \multicolumn{2}{|c|}{ Men, no. $(\%)^{\star}$} & \multicolumn{2}{|c|}{ Women, no. (\%)* } \\
\hline & $\begin{array}{c}\text { Dermatomyositis } \\
\qquad n=514\end{array}$ & $\begin{array}{l}\text { Control } \\
n=2480\end{array}$ & $\begin{array}{c}\text { Dermatomyositis } \\
\qquad n=1088\end{array}$ & $\begin{array}{l}\text { Control } \\
n=5501\end{array}$ \\
\hline Age, yr, mean \pm SD & $49.45 \pm 15.32$ & $48.45 \pm 15.35$ & $47.44 \pm 14.37$ & $46.86 \pm 14.09$ \\
\hline \multicolumn{5}{|l|}{ Age group, yr } \\
\hline$\geq 20,<30$ & 64 (12.45) & 348 (14.03) & 137 (12.59) & 708 (12.87) \\
\hline$\geq 30,<40$ & $67(13.04)$ & 345 (13.91) & $180(16.54)$ & 956 (17.38) \\
\hline$\geq 40,<50$ & $117(22.76)$ & $569(22.94)$ & $298(27.39)$ & $1522(27.67)$ \\
\hline$\geq 50,<60$ & $136(26.46)$ & $624(25.16)$ & $252(23.16)$ & 1285 (23.36) \\
\hline$\geq 60,<70$ & 75 (14.59) & $355(14.31)$ & $141(12.96)$ & $684(12.43)$ \\
\hline$\geq 70,<80$ & $47(9.14)$ & 207 (8.35) & $71(6.53)$ & $310(5.64)$ \\
\hline$\geq 80$ & $8(1.56)$ & 32 (1.29) & $9(0.83)$ & $36(0.65)$ \\
\hline$p$ value & \multicolumn{2}{|c|}{0.9} & \multicolumn{2}{|c|}{0.9} \\
\hline
\end{tabular}

Table 2: Age-adjusted standardized incidence ratio of Sjögren syndrome in patients with dermatomyositis, stratified by age

\section{Dermatomyositis \\ $n=1602$ \\ Control \\ $n=7981$}

No. (incidence)*

20-29

$21(19.37)$

30-39

$22(17.33)$

$43(20.78)$

$39(22.23)$

9 (9.55)

60-69

$5(11.13)$

Total

139 (18.37)

Age-adjusted standardized incidence ratio

Note: $\mathrm{Cl}=$ confidence interval.

${ }^{\star}$ Numbers of newly diagnosed Sjögren syndrome (incidence rate per 1000 person-years).

†The total follow-up period (person-years).

\begin{tabular}{cccc} 
Follow-up $\dagger$ & No. (incidence) & Follow-up & Rate ratio $(95 \%$ CI) \\
\hline 1084.00 & $23(3.72)$ & 6189.53 & $5.21(2.89-9.42)$ \\
\hline 1269.83 & $20(2.52)$ & 7949.00 & $6.89(3.76-12.62)$ \\
\hline 2068.83 & $40(3.54)$ & 11306.67 & $5.88(3.82-9.04)$ \\
\hline 1754.50 & $27(3.03)$ & 8902.50 & $7.33(4.49-11.97)$ \\
\hline 942.00 & $14(3.11)$ & 4508.67 & $3.08(1.33-7.11)$ \\
\hline 449.33 & $3(1.56)$ & 1924.17 & $7.14(1.71-29.87)$ \\
\hline 7568.50 & $127(3.11)$ & 40780.58 & $5.90(4.64-7.50)$ \\
& & & $5.92(3.82-9.16)$ \\
\hline
\end{tabular}


age, sex and other concomitant autoimmune diseases (rheumatoid arthritis, systemic lupus erythematosus and systemic sclerosis) (Table 5). There appeared to be a significant correlation between Sjögren syndrome and male sex with dermatomyositis, as evidenced by a higher hazard ratio when compared with controls (HR 2.69, 95\% Cl 1.19-6.09) (Table 5).

\section{Cumulative incidences of Sjögren syndrome}

Overall, patients with dermatomyositis had a higher incidence of Sjögren syndrome when compared with the control group $(p<$ 0.001 ) (data not shown). When stratified by sex, a similar result was seen. The dermatomyositis group was associated with a higher cumulative incidence of Sjögren syndrome than the control group in both men $(p<0.001)$ and women $(p<0.001)$ (Figure 1$)$.

\section{Interpretation}

We have shown a higher incidence of Sjögren syndrome among patients with dermatomyositis than among those without. When stratified by age and sex, similar trends persisted. A history of der- matomyositis was significantly associated with subsequent Sjögren syndrome, even after adjusting for age, sex and concomitant rheumatoid arthritis, systemic lupus erythematosus and systemic sclerosis. There was also a strong interaction between dermatomyositis and being male.

Sjögren syndrome has important implications for dermatomyositis patients and the physicians guiding their treatment. Reportedly, phenotypes (e.g., myositis) may be altered in patients with concurrent anti-SSA antibodies, hallmarks of Sjögren syndrome. ${ }^{13}$ These alterations include perivascular inflammation, ${ }^{15}$ myositis deterioration, malignant disease, ${ }^{32}$ left ventricular diastolic dysfunction, ${ }^{33}$ interstitial lung disease ${ }^{34}$ and death. ${ }^{32}$ Sjögren syndrome combined with other autoimmune diseases was associated with increased T-cell activation, ${ }^{35}$ Raynaud phenomenon, ${ }^{36}$ thyroid disease, ${ }^{37}$ cardiac electrical instability ${ }^{38}$ and lymphoma, ${ }^{39}$ more so than with the respective autoimmune diseases alone. Thus, Sjögren syndrome in dermatomyositis potentially defines a distinct disease subset of dermatomyositis. Considering the substantial risk of Sjögren syndrome in dermatomyositis as shown in this study, studies to clarify whether Sjögren syndrome associated

Table 3: Age-adjusted standardized incidence ratio of Sjögren syndrome in male patients with dermatomyositis, stratified by age

\section{Dermatomyositis \\ $n=514$}

\section{Control}

$n=2480$

$\begin{array}{lccccc}\text { Age, } \mathbf{y r} & \text { No. (incidence)* } & \text { Follow-upt } & \text { No. (incidence) } & \text { Follow-up } & \text { Rate ratio (95\% CI) } \\ 20-39 & 4(6.28) & 637.08 & 2(0.52) & 3839.58 & 12.05(2.21-65.81) \\ 40-59 & 13(11.58) & 1122.33 & 5(0.91) & 5484.42 & 12.71(4.53-35.64) \\ \geq 60 & 5(10.59) & 472.25 & 2(0.90) & 2231.25 & 11.81(2.29-60.88) \\ \text { Total } & 22(9.86) & 2231.67 & 9(0.78) & 11555.25 & 12.66(5.83-27.49) \\ \text { Age-adjusted standardized incidence ratio } & & & & 12.37(2.68-57.02)\end{array}$

Note: $\mathrm{Cl}=$ confidence interval.

${ }^{\star}$ Numbers of newly diagnosed Sjögren syndrome (incidence rate per 1000 person-years).

†The total follow-up period (person-years).

Table 4: Age-adjusted standardized incidence ratio of Sjögren syndrome in female patients with dermatomyositis, stratified by age
Dermatomyositis
$n=1088$
Control
$n=5501$

\begin{tabular}{|c|c|c|c|c|c|}
\hline Age, yr & No. (incidence)* & Follow-upt & No. (incidence) & Follow-up & Rate ratio $(95 \% \mathrm{Cl})$ \\
\hline $20-29$ & $20(26.19)$ & 763.67 & $22(5.25)$ & 4188.25 & $4.99(2.72-9.14)$ \\
\hline $30-39$ & 19 (19.94) & 953.08 & $19(3.11)$ & 6110.75 & $6.41(3.39-12.11)$ \\
\hline $40-49$ & $38(24.74)$ & 1535.75 & $36(4.29)$ & 8397.50 & $5.77(3.66-9.11)$ \\
\hline $50-59$ & $31(26.60)$ & 1165.25 & $26(4.11)$ & 6327.25 & $6.47(3.84-10.90)$ \\
\hline $60-69$ & $6(9.24)$ & 649.67 & $13(4.32)$ & 3008.58 & $2.14(0.81-5.62)$ \\
\hline$\geq 70$ & $3(11.14)$ & 269.42 & $2(1.68)$ & 1193.00 & $6.64(1.11-39.75)$ \\
\hline Total & $117(21.92)$ & 5336.83 & $118(4.04)$ & 29225.33 & $5.43(4.20-7.01)$ \\
\hline \multicolumn{5}{|c|}{ Age-adjusted standardized incidence ratio } & $5.42(3.42-8.57)$ \\
\hline
\end{tabular}


with dermatomyositis might have independent prognostic importance are needed.

Although we did not explore the mechanism of association between dermatomyositis and Sjögren syndrome, plausible explanations may include shared serological, genetic and autoantibody components, namely interleukin-4 (IL-4), B-cell activating factor (BAFF), IRF5 rs4728142 and anti-SSA. Serum IL-4 is increased in both patients with dermatomyositis ${ }^{40}$ and those with Sjögren syndrome, ${ }^{41}$ and IL-4 knockout mice lack immune-mediated secretory dysfunction. ${ }^{42}$ Moreover, there was increased BAFF in both patients with dermatomyositis ${ }^{43}$ and those with Sjögren syndrome. ${ }^{44}$ In patients with Sjögren syndrome, BAFF levels correlated with Sjögren

\begin{tabular}{|c|c|c|}
\hline Variable & $\begin{array}{l}\text { Adjusted HR } \\
(95 \% \mathrm{CI})\end{array}$ & $p$ value \\
\hline Age & $1.00(0.99-1.01)$ & 0.9 \\
\hline Female sex & $3.92(1.98-7.75)$ & $<0.001$ \\
\hline Dermatomyositis & $2.67(2.01-3.54)$ & $<0.001$ \\
\hline $\begin{array}{l}\text { Interaction term (male } \\
\text { dermatomyositis) }\end{array}$ & $2.69(1.19-6.09)$ & 0.02 \\
\hline Rheumatoid arthritis & $3.79(2.92-4.92)$ & $<0.001$ \\
\hline Systemic lupus erythematosus & $3.97(2.99-5.28)$ & $<0.001$ \\
\hline Systemic sclerosis & $1.53(1.03-2.28)$ & 0.03 \\
\hline
\end{tabular}

A

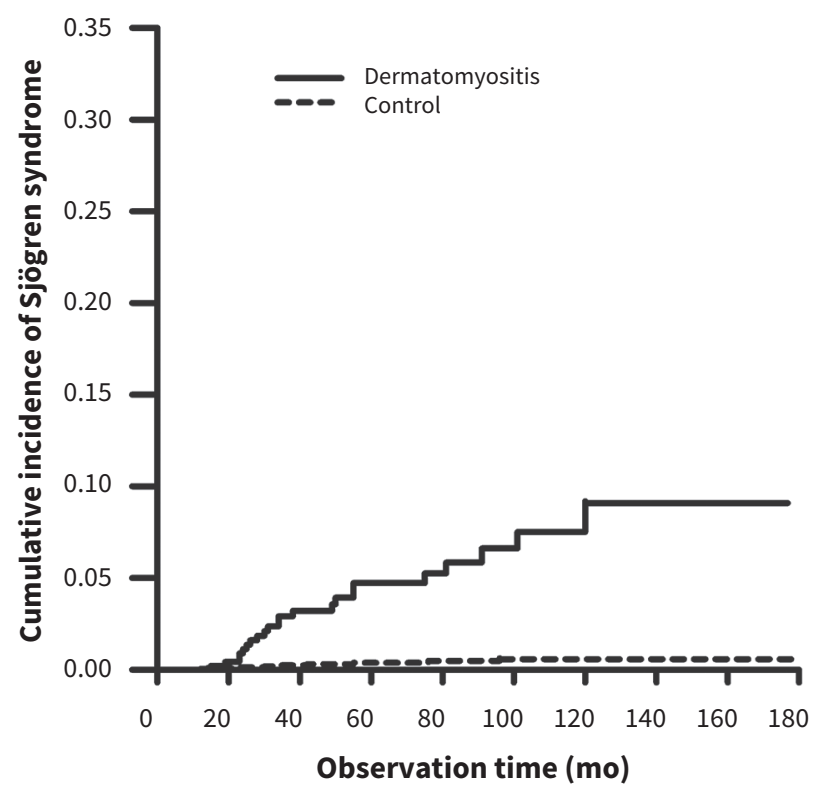

syndrome Disease Damage Index. ${ }^{44}$ In mice, BAFF overexpression aggravated salivary gland inflammation (sialoadenitis) and increased Sjögren syndrome..$^{45}$ Conversely, BAFF receptor blockade decreased autoantibodies and sialoadenitis. ${ }^{46}$ Furthermore, the IRF5 rs4728142 variant was associated with both dermatomyositis ${ }^{17}$ and Sjögren syndrome. ${ }^{18}$ Moreover, dermatomyositis is associated with a high frequency of anti-SSA, ${ }^{15}$ which increased risk of Sjögren syndrome. ${ }^{47}$ Thus, common cytokines (IL-4, BAFF), gene polymorphism (IRF5 rs4728142) and autoantibodies (anti-SSA) probably underpin the link between dermatomyositis and Sjögren syndrome.

Although the incidence of Sjögren syndrome in this study was higher overall in women than in men, the incidence rate ratio for the male cohort was higher than that of the female cohort. The differential association of Sjögren syndrome between male and female patients with dermatomyositis may be explained by the different pathogenesis of dermatomyositis in the different sexes. Past studies of dermatomyositis showed sex differences in risk factor ${ }^{22}$ and autoantibody profiles..$^{24}$ Furthermore, genetic studies of pediatric dermatomyositis showed sexual dimorphism in the gene-gene interaction. ${ }^{48}$ These data indicated distinct immunopathologic mechanisms of dermatomyositis between men and women. Alternatively, it is also possible that dermatomyositis changes whatever exists in men that protects them from developing Sjögren syndrome. For example, dermatomyositis exerted sex differential effects on expression of S100A4, a protein implicated in inflammatory and autoimmune diseases. ${ }^{49}$ Future studies may help address these possibilities.

\section{Strengths and limitations}

The population-based nature of the cohort, the sample size from actual clinical settings, and the validated approach to data gathering strengthened the reliability of this study.

\section{B}

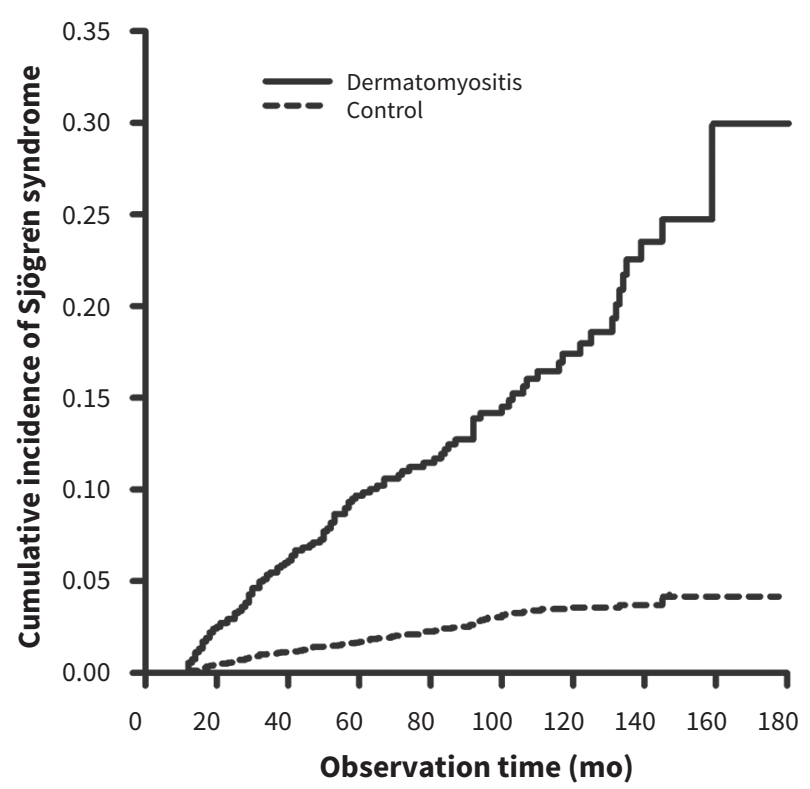

Figure 1: Cumulative incidence rates of Sjögren syndrome among patients in the dermatomyositis and control groups in both (A) men and (B) women (both $p<0.001$, estimated by the log-rank test). 
Misclassification cannot be totally excluded in the administrative databases. However, inclusion of any cases that were not Sjögren syndrome was not differential, and thus would decrease the likelihood that changes in rates would be detected. Therefore, misclassification would bias toward underestimation, rather than overestimation, of the association between dermatomyositis and Sjögren syndrome.

Retrospective cohort study designs raise concerns that differences in delay to diagnosis between patients with dermatomyositis and control patients might play a role in the connection between dermatomyositis and Sjögren syndrome. However, past studies showed similar delays to diagnosis of Sjögren syndrome between patients with and without antecedent autoimmune diseases. ${ }^{31}$ Accordingly, differences in delay to diagnosis is unlikely to account for our findings.

Because clinical phenotypes or serologic characteristics of dermatomyositis were unavailable in administrative databases, whether patients with dermatomyositis positive for anti-SSA were those who presented with Sjögren syndrome was unknown. Further investigation to clarify the incidence of Sjögren syndrome in dermatomyositis in regard to phenotypes or serologic characteristics is warranted.

Finally, although patients with Sjögren syndrome had an increased risk of non-Hodgkin lymphoma, ${ }^{50}$ administrative data limited our ability to assess whether patients with dermatomyositis and Sjögren syndrome had increased risk of malignant disease, particularly non-Hodgkin lymphoma, and the possible role of anti-transcription intermediary factor $1 \gamma$ (anti-155/140) antibodies $^{51}$ as a serological marker for malignant disease. ${ }^{52}$ This question should be addressed by future studies.

\section{Conclusion}

This nationwide retrospective cohort study sheds light on the sex differential association of Sjögren syndrome in patients with dermatomyositis. Because treatment in the early phases of Sjögren syndrome shows a better response,,$^{53,54}$ early recognition of the condition is important. Future studies should clarify the meaning of Sjögren syndrome in dermatomyositis by investigating the association of Sjögren syndrome to clinical outcomes within a dermatomyositis cohort and identifying the contributions of different autoantibodies to the risks of Sjögren syndrome in these patients. In addition, the relationship between dermatomyositis and Sjögren syndrome could suggest a common pathophysiology in these 2 diseases. Mechanistic studies of sex differential association of dermatomyositis with Sjögren syndrome could provide new targets of therapy.

\section{References}

1. Avina-Zubieta JA, Sayre EC, Bernatsky S, et al. Adult prevalence of systemic autoimmune rheumatic diseases (SARDs) in British Columbia, Canada [abstract]. Arthritis Rheum 2011;63(Suppl 10):1846.

2. Kuo CF, See LC, Yu KH, et al. Incidence, cancer risk and mortality of dermatomyositis and polymyositis in Taiwan: a nationwide population study. Br J Dermatol 2011;165:1273-9.

3. Marie I. Morbidity and mortality in adult polymyositis and dermatomyositis. Curr Rheumatol Rep 2012;14:275-85.

4. Rai SK, Choi HK, Sayre EC, et al. Risk of myocardial infarction and ischaemic stroke in adults with polymyositis and dermatomyositis: a general populationbased study. Rheumatology (Oxford) 2016;55:461-9.
5. Chen YJ, Wu CY, Shen JL. Predicting factors of interstitial lung disease in dermatomyositis and polymyositis. Acta Derm Venereol 2007;87:33-8.

6. Bettero RG, Cebrian RF, Skare TL. Prevalence of ocular manifestation in 198 patients with rheumatoid arthritis: a retrospective study. Arq Bras Oftalmol 2008;71:365-9.

7. Chambers SA, Charman SC, Rahman A, et al. Development of additional autoimmune diseases in a multiethnic cohort of patients with systemic lupus erythematosus with reference to damage and mortality. Ann Rheum Dis 2007; 66:1173-7.

8. Muangchan C; Canadian Scleroderma Research Group. Baron M, Pope J. The $15 \%$ rule in scleroderma: the frequency of severe organ complications in systemic sclerosis. A systematic review. J Rheumatol 2013;40:1545-56.

9. Hatron PY, Wallaert B, Fourrier JL, et al. Dermatopolymyositis and pulmonary fibrosis associated with Gougerot-Sjögren syndrome. Study of 3 cases [article in French]. Rev Med Interne 1985;6:97-103.

10. Huang CT, Chen YC, Lin C, et al. A case of dermatomyositis with secondary Sjögren's Syndrome-diagnosis with follow-up study of technetium-99m pyrophosphate scintigraphy. Acta Neurol Taiwan 2013;22:162-8.

11. Ortigosa LC, Reis VM. Dermatomyositis: analysis of 109 patients surveyed at the Hospital das Clínicas (HCFMUSP), São Paulo, Brazil. An Bras Dermatol 2014;89:719-27.

12. Fiorentino DF, Presby M, Baer AN, et al. PUF60: a prominent new target of the autoimmune response in dermatomyositis and Sjögren's syndrome. Ann Rheum Dis 2016;75:1145-51.

13. Mavragani CP, Moutsopoulos HM. Sjögren syndrome. CMAJ 2014;186:E579-86.

14. Koler RA, Montemarano A. Dermatomyositis. Am Fam Physician 2001;64:1565-72.

15. Pinal-Fernandez I, Casciola-Rosen LA, Christopher-Stine L, et al. The prevalence of individual histopathologic features varies according to autoantibody status in muscle biopsies from patients with dermatomyositis. J Rheumatol 2015;42:1448-54.

16. Rutjes SA, Vree Egberts WT, Jongen P, et al. Anti-Ro52 antibodies frequently cooccur with anti-Jo-1 antibodies in sera from patients with idiopathic inflammatory myopathy. Clin Exp Immunol 1997;109:32-40.

17. Chen S, Wang Q, Wu Z, et al. Genetic association study of TNFAIP3, IFIH1, IRF5 polymorphisms with polymyositis/dermatomyositis in Chinese Han population. PLoS One 2014;9:e110044.

18. Lessard CJ, Li H, Adrianto I, et al. Variants at multiple loci implicated in both innate and adaptive immune responses are associated with Sjögren's syndrome. Nat Genet 2013;45:1284-92.

19. Brito-Zerón P, Kostov B, Solans R, et al. Systemic activity and mortality in primary Sjögren syndrome: predicting survival using the EULAR-SS Disease Activity Index (ESSDAI) in 1045 patients. Ann Rheum Dis 2016;75:348-55.

20. Callaghan R, Prabu A, Allan RB, et al. Direct healthcare costs and predictors of costs in patients with primary Sjögren's syndrome. Rheumatology (Oxford) 2007;46:105-11.

21. Bowman SJ, St Pierre Y, Sutcliffe N, et al. Estimating indirect costs in primary Sjögren's syndrome. J Rheumatol 2010;37:1010-5.

22. Love LA, Weinberg CR, McConnaughey DR, et al. Ultraviolet radiation intensity predicts the relative distribution of dermatomyositis and anti-Mi-2 autoantibodies in women. Arthritis Rheum 2009;60:2499-504.

23. Olazagasti JM, Hein M, Crowson CS, et al. Adipokine gene expression in peripheral blood of adult and juvenile dermatomyositis patients and their relation to clinical parameters and disease activity measures. J Inflamm (Lond) 2015; 12:29.

24. Hamaguchi Y, Kuwana M, Hoshino K, et al. Clinical correlations with dermatomyositis-specific autoantibodies in adult Japanese patients with dermatomyositis: a multicenter cross-sectional study. Arch Dermatol 2011;147:391-8.

25. Universal health coverage in Taiwan. Taiwan: Bureau of National Health Insurance, Department of Health, Executive Yuan; 2012. Available: www.nhi.gov.tw/ Resource/webdata/21717_1_20120808UniversalHealthCoverage.pdf (accessed 2016 May 28).

26. Yang NP, Chen HC, Phan DV, et al. Epidemiological survey of orthopedic joint dislocations based on nationwide insurance data in Taiwan, 2000-2005. BMC Musculoskelet Disord 2011;12:253.

27. Bohan A, Peter JB. Polymyositis and dermatomyositis. N Engl J Med 1975;292: 344-7, 403-7.

28. Wu PH, Lin YT, Yang YH, et al. The increased risk of active tuberculosis disease in patients with dermatomyositis - a nationwide retrospective cohort study. Sci Rep 2015;5:16303.

29. Kuo CF, Grainge MJ, Valdes AM, et al. Familial risk of Sjögren's syndrome and coaggregation of autoimmune diseases in affected families: a nationwide population study. Arthritis Rheumatol 2015;67:1904-12.

30. Wu CS, Gau SS, Lai MS. Long-term antidepressant use and the risk of type 2 diabetes mellitus: a population-based, nested case-control study in Taiwan. J Clin Psychiatry 2014;75:31-8. 
31. Rasmussen A, Radfar L, Lewis D, et al. Previous diagnosis of Sjögren's syndrome as rheumatoid arthritis or systemic lupus erythematosus. Rheumatology (Oxford) 2016;55:1195-201.

32. Marie I, Hatron PY, Dominique S, et al. Short-term and long-term outcome of anti-Jo1-positive patients with anti-Ro52 antibody. Semin Arthritis Rheum 2012;41:890-9.

33. Diederichsen LP, Simonsen JA, Diederichsen AC, et al. Cardiac abnormalities in adult patients with polymyositis or dermatomyositis as assessed by non-invasive modalities. Arthritis Care Res (Hoboken) 2016;68:1012-20.

34. Srivastava P, Dwivedi S, Misra R. Myositis-specific and myositis-associated autoantibodies in Indian patients with inflammatory myositis. Rheumatol Int 2016;36:935-43.

35. Hadjinicolaou AV, Wu L, Fang B, et al. Relationship of CD146 expression to activation of circulating T cells: exploratory studies in healthy donors and patients with connective tissue diseases. Clin Exp Immunol 2013;174:73-88.

36. Baer AN, Maynard JW, Shaikh F, et al. Secondary Sjögren's syndrome in systemic lupus erythematosus defines a distinct disease subset. J Rheumatol 2010;37:1143-9.

37. Scofield RH, Bruner GR, Harley JB, et al. Autoimmune thyroid disease is associated with a diagnosis of secondary Sjögren's syndrome in familial systemic lupus. Ann Rheum Dis 2007;66:410-3.

38. Pirildar T, Sekuri $C$, Utük $O$, et al. QT dispersion in rheumatoid arthritis patients with and without Sjögren's syndrome. Clin Rheumatol 2003;22:225-8.

39. Baldini C, Mosca M, Della Rossa A, et al. Overlap of ACA-positive systemic sclerosis and Sjögren's syndrome: a distinct clinical entity with mild organ involvement but at high risk of lymphoma. Clin Exp Rheumatol 2013;31:272-80.

40. Shimojima Y, Ishii W, Matsuda M, et al. Phenotypes of peripheral blood lymphocytes and cytokine expression in polymyositis and dermatomyositis before treatment and after clinical remission. Clin Med Insights Arthritis Musculoskelet Disord 2012;5:77-87.

41. Wu GL, Li TY, Fan YS, et al. Effect of Chinese herbal medicine for nourishing yin, supplementing qi, and activating blood on the Th1/Th2 immune balance in peripheral blood in patients with primary Sjögren's syndrome. Chin J Integr Med 2013;19:696-700.

42. Gao J, Killedar S, Cornelius JG, et al. Sjögren's syndrome in the NOD mouse model is an interleukin-4 time-dependent, antibody isotype-specific autoimmune disease. J Autoimmun 2006;26:90-103.
43. Krystufková $\mathrm{O}$, Vallerskog $\mathrm{T}$, Helmers $\mathrm{SB}$, et al. Increased serum levels of B cell activating factor (BAFF) in subsets of patients with idiopathic inflammatory myopathies. Ann Rheum Dis 2009;68:836-43.

44. Vadacca M, Margiotta D, Sambataro D, et al. BAFF/APRIL pathway in Sjögren syndrome and systemic lupus erythematosus: relationship with chronic inflammation and disease activity. Reumatismo 2010;62:259-65.

45. Groom J, Kalled SL, Cutler AH, et al. Association of BAFF/BLyS overexpression and altered B cell differentiation with Sjögren's syndrome. J Clin Invest 2002; 109:59-68.

46. Sharma A, Kiripolsky J, Klimatcheva E, et al. Early BAFF receptor blockade mitigates murine Sjögren's syndrome: concomitant targeting of CXCL13 and the BAFF receptor prevents salivary hypofunction. Clin Immunol 2016;164:85-94.

47. Jonsson R, Theander E, Sjöström B, et al. Autoantibodies present before symptom onset in primary Sjögren syndrome. JAMA 2013;310:1854-5.

48. Niewold TB, Kariuki SN, Morgan GA, et al. Gene-gene-sex interaction in cytokine gene polymorphisms revealed by serum interferon alpha phenotype in juvenile dermatomyositis. J Pediatr 2010;157:653-7.

49. Pleštilová L, Mann H, Andrés Cerezo L, et al. The metastasis promoting protein S100A4 levels associate with disease activity rather than cancer development in patients with idiopathic inflammatory myopathies. Arthritis Res Ther 2014;16:468.

50. Engels EA, Parsons R, Besson C, et al. Comprehensive evaluation of Medical Conditions Associated with Risk of Non-Hodgkin Lymphoma using Medicare Claims ("MedWAS"). Cancer Epidemiol Biomarkers Prev 2016;25:1105-13.

51. Fujimoto M, Hamaguchi Y, Kaji K, et al. Myositis-specific anti-155/140 autoantibodies target transcription intermediary factor 1 family proteins. Arthritis Rheum 2012;64:513-22.

52. Ikeda N, Takahashi K, Yamaguchi Y, et al. Analysis of dermatomyositis-specific autoantibodies and clinical characteristics in Japanese patients. J Dermatol 2011;38:973-9.

53. Pijpe J, van Imhoff GW, Spijkervet FK, et al. Rituximab treatment in patients with primary Sjögren's syndrome: an open-label phase II study. Arthritis Rheum 2005; 52:2740-50.

54. Devauchelle-Pensec V, Pennec Y, Morvan J, et al. Improvement of Sjögren's syndrome after two infusions of rituximab (anti-CD20). Arthritis Rheum 2007; 57:310-7.

\section{Competing interests: None declared.}

This article has been peer reviewed.

Affiliations: Department of Internal Medicine (Tseng), Kaohsiung Municipal Ta-Tung Hospital; Department of Kinesiology (Chang), Health and Leisure Studies, National University of Kaohsiung; Division of Rheumatology (Tsai, Ou, Wu, Sung, Yen), Department of Internal Medicine, Kaohsiung Medical University Hospital; Graduate Institute of Medicine (Sung, Yen), College of Medicine, Kaohsiung Medical University; Division of Endocrinology and Metabolism, Department of Internal Medicine (Hsieh), Changhua Christian Hospital, Changhua, Taiwan; Graduate Institute of Integrated Medicine (Hsieh), China Medical University, Taichung, Taiwan
Contributors: Chia-Chun Tseng, Shun-Jen Chang, and Jeng-Hsien Yen contributed to the conception and design of the study. Shun-Jen Chang and Ming-Chia Hsieh were involved in the acquisition of data. Wen-Chan Tsai, Tsan-Teng Ou, Cheng-Chin Wu, and Wan-Yu Sung were involved in the analysis and interpretation of the data. Chia-Chun Tseng, Shun-Jen Chang, and Jeng-Hsien Yen drafted the manuscript. All of the authors reviewed the manuscript critically for intellectual content and approved the final version to be published.

Acknowledgement: The authors thank all of the patients enrolled in Taiwan's National Health Insurance Research Database and Registry of Catastrophic Illness database.

Accepted: Sept. 29, 2016

Correspondence to: Jeng-Hsien Yen, jehsye@kmu.edu.tw 